Relations industrielles

Industrial Relations

\title{
Les réformes de structure
}

\section{André Roy}

Volume 4, numéro 8, avril 1949

URI : https://id.erudit.org/iderudit/1023516ar

DOI : https://doi.org/10.7202/1023516ar

Aller au sommaire du numéro

Éditeur(s)

Département des relations industrielles de l’Université Laval

ISSN

0034-379X (imprimé)

1703-8138 (numérique)

Découvrir la revue

Citer cet article

Roy, A. (1949). Les réformes de structure. Relations industrielles / Industrial

Relations, 4(8), 78-79. https://doi.org/10.7202/1023516ar

Tous droits réservés @ C Département des relations industrielles de l’Université Laval, 1949
Ce document est protégé par la loi sur le droit d'auteur. L'utilisation des services d'Érudit (y compris la reproduction) est assujettie à sa politique d'utilisation que vous pouvez consulter en ligne.

https://apropos.erudit.org/fr/usagers/politique-dutilisation/ 


\section{LE DÉLAI-CONGÉ}

Le législateur à la fin du siècle dernier formula des règles sur la rupture du contrat de travail; elles se trouvent à la Loi des maîtres et des serviteurs (S.R.Q., 1941, c. 328). Suivant ce statut, le délai-congé pouvait être arrêté dans les cités et villes, par un règlement municipal et ailleurs l'était par les dispositions de cette loi.

Elle visait les domestiques, serviteurs, compagnons et journaliers, en un mot, les ouvriers; elle s'appliquait à ceux qui s'étaient engagés pour une période indéterminée. Leur contrat de travail ne pouvait être rompu de part et d'autre sans un avis de délai-congé.

Mais les dispositions de cette loi ne répondaient plus à notre éthique sociale, car elle prévoyait l'amende pour l'abandon du travail sans avis. De nombreuses bonnes et apprenties durent affronter les cours pour avoir laissé en plan leurs employeurs.

Le législateur à la dernière session par le statut 13 Geo. VI, c. 69 a abrogé la Loi des maîtres et des serviteurs ainsi que les règlements municipaux adoptés sous son empire. La Loi de l'engagement des pêcheurs, (S.R.Q., 1941, c. 329), statut au même effet, fut également abrogée.

Le paragraphe suivant fut introduit au Code civil à l'article 1668:

«Dans le cas d'un domestique, serviteur, compagnon ou journalier engagé à la semaine, au mois ou à l'année, mais pour un laps de temps indéfini, il peut être mis fin au contrat par avis, de l'une des parties à l'autre, d'une semaine, si l'engagement est à la semaine; de deux semaines, si l'engagement est au mois; d'un mois, si l'engagement est à l'année. »

Ce nouvel article fixe suivant le mode de paye la longueur du délai-congé, explicitant la règle des articles 1642 et 1657 du Code civil applicable aux autres salariés engagés pour une durée non déterminée.

\section{LES RÉFORMES DE STRUCTURE}

Depuis quelque temps, on parle de réformes de l'entreprise dans beaucoup de milieux.

Les uns prennent immédiatement la mouche, crient à la révolution ou au renversement de l'ordre établi alors qu'à peine commence le cheminement de ces idées contenues en principe dans les encycliques sociales des souverains pontifes.

Les autres - et c'est heureusement le cas de la majorité, encore qu'ils pétaradent moins haut, - d'un jugement moins superficiel s'efforcent plutôt d'étudier objectivement ces données nouvelles afin de voir s'il est possible, par une évolution à la fois prudente et audacieuse, d'en assurer l'application raisonnable de manière à purger l'économie moderne des abus d'un capitalisme anonyme et toutpuissant.

Telle est la première constatation qui vient à l'esprit de celui qui se donne la peine de parcourir attentivement une brochure publiée il y a quelques jours à peine, en français et en anglais, par le Département des relations industrielles de la Faculté des sciences sociales de l'Université Laval sous la signature de MM. les abbés Gérard Dion et Paul-Emile Bolté ainsi que de Marcel Clément. Cette brochure s'intitule: «Réformes de structure dans l'entreprise ». ${ }^{1}$

(1) «Réformes de structure dans l'entreprise » est en vente au Département des relations industrielles de l'Université Laval, Québec, au prix de \$1.00.
Dans une introduction fort à point, M. l'abbé Gérard Dion pose froidement le problème en rappelant qu'il y a déjà seize ans, dans l'encyclique Quadragesimo Anno, Sa Sainteté Pie XI lançait le mot d'ordre: Réforme des moeurs, réformes des institutions.

«Les réformes de structures, dans le domaine des relations du travail, écrit-il, se posent sur le plan de lentreprise, de la profession et de la nation. Elles ont pour base le problème de la propriété et de sa distribution sous toutes ses formes. Cependant, on ne peut pas oublier non plus que l'enjeu du conflit entre les patrons et les ouvriers n'est pas seulement une question de piastres et de cents, mais une répartition nouvelle de l'autorité et une reconnaissance pratique de la dignité humaine. Voilà pourquoi, nous avons l'intention, sans courir l'aventure dans les fossés de gauche et de droite mais en nous basant sur la doctrine sociale de l'Eglise, qui est assez féconde par elle-même, et sur les réalités sociales telles qu'elles se présentent chez nous, de repenser les conceptions que l'on se fait des régimes de la propriété, de l'entreprise, du capitalisme, du profit, de l'autorité dans les relations du travail, de la participation à la propriété, à la gestion et au profit de l'entreprise. »

Les études de la brochure portent sur les réformes de structure au sein de l'entreprise. C'est la base. Inutile, en effet, de songer à réformer la profession et, encore moins, l'économie dans son ensemble, si les fondements font défaut, s'ils ne s'adaptent plus à la condition actuelle des employeurs et des salariés, si la rupture est totale 
entre le capital et le travail, si l'économique et le social sont placés, par l'appareil juridique qui les encadre, dans l'impossibilité de se rencontrer et de se compénétrer.

A sa base même, la réforme de l'entreprise accroche le problème de la propriété. Il est, à ce sujet, étonnant de remarquer combien habile fut, depuis un siècle, le régime capitaliste, à se faire passer comme le défenseur incontesté, l'unique gardien de la propriété personnelle, combien il fut habile, loup revêtant la peau de l'agneau, à se confondre avec elle, à se faire pur, si l'on peut ainsi s'exprimer, alors qu'en réalité, dans quatre-vingt-dix pour cent des grandes organisations commerciales et industrielles, il représentent ce qui peut exister de plus anonyme, de plus collectif, de plus diffus.

Dans un chapitre intitulé «Propriété et entreprise», M. l'abbé Bolté définit la propriété, énumère ses titres, fait ensuite les applications qui s'imposent à l'entreprise dont les modalités peuvent varier de la propriété personnelle à un rassemblement de capitaux venant des cinq parties du monde. L'auteur conclut de tout ceci, que, d'une part «sous l'inspiration du libéralisme économique, l'argent règne en maître, qu'on délaisse la fonction sociale inhérente à la propriété, le service inhérent à l'autorité », et que, d'autre part, si l'on veut corriger cet état de choses, faire de la grande entreprise \&une réalité communautaire », il faut tempérer le contrat de salariat par des éléments empruntés au contrat de société.

De son côté, M. Marcel Clément, après avoir opposé la libre entreprise à l'entreprise libérale, fait les distinctions nécessaires entre la paternité, le patronat et le paternalisme, analyse la structure de l'entreprise moderne, explique quelques-unes des solutions qui peuvent s'appliquer pour corriger ses défauts et combler ses lacunes. M. l'abbé Gérard Dion étudie la moralité de la participation aux bénéfices tandis que $M$. Clément laisse entrevoir le rôle que doit jouer le syndicalisme dans l'élaboration des réformes de structure.

Tous ceux qui veulent réellement et sincèrement travailler à la restauration de l'ordre social trouveront porfit à lire et à étudier la brochure « Réformes de structure dans l'entreprise ». Même si elle ne vide pas la question, comme le font remarquer ses auteurs, elle contribuera singulièrement à renseigner, à faire réfléchir et à ouvrir des horizons nouveaux.

(L'Action Catholique)

André Roy

\section{LA PROCÉDURE D'ARBITRAGE \\ Son usage dans la province de Québec}

On consultera avec beaucoup d'intérêt le rapport annuel du ministère du Travail pour l'année 1947-48. Des statistiques ont été compilées qui permettent au lecteur de se rendre compte de l'immense effort de conciliation et d'arbitrage qui a été donné sans bruit ni grande publicité par les conciliateurs et les arbitres désignés par l'autorité publique.

Et d'abord, un coup d'oeil rapide sur le rapport de la Commission de relations ouvrières nous indique qu'au cours de cette année les syndiqués ont déposé 676 conventions collectives couvrant 158,317 salariés. Un rapport complémentaire indique qu'en tenant compte des conventions terminées, dénoncées et non renouvelées, on constate que 1,136 conventions sont en vigueur couvrant 163,548 salariés.

Nous ne sommes pas en mesure d'expliquer le décalage considérable entre le nombre des conventions déposées au cours de l'année et celui des conventions actuellement en vigueur comme il nous est impossible d'expliquer la faible différence entre 158,317 salariés couverts par les conventions de l'année actuelle et 163,548 salariés couverts par les conventions actuellement en vigueur. A tout événement, on peut trouver ces chiffres à la page $33 \mathrm{du}$ rapport annuel.
Le tableau 9, aux pages 34 et suivantes, classe par affiliation syndicale le nombre de conventions déposées ainsi que les employés couverts durant la même année.

La Confédération des Travailleurs catholiques du Canada, inc., a à son crédit 163 conventions couvrant 44,057 salariés. 124 de ces conventions ont été conclues à la suite de négociations directes, ce qui constitue un très fort pourcentage. Par contre 39 sont passées par le stade de la conciliation et 13 par celui de l'arbitrage.

Les syndicats indépendants incorporés ou non ont à leur crédit 73 conventions couvrant 27,175 salariés; 66 de ces conventions ont été conclues par négociations directes, 7 l'ont été au stade de la conciliation et 3 seulement à celui de l'arbitrage.

Quant au Congrès canadien du Travail il compte 71 conventions intéressant 12,873 salariés. De ces conventions, 50 ont été négociées directement par les intéressés, 20 ont subi le stade de la conciliation et 14 celui de l'arbitrage.

Enfin, le Congrès des Métiers et du Travail auquel sont affiliées les unions de la Fédération américaine du Travail, a à son crédit 137 conventions visant 40,280 salaries. De ces conventions, 110 ont été signées à la suite de négociations di- 